\title{
Las comunidades de briófitos y su relacion con la estructura de la vegetaticón fanerogamica, en el gradiente altitudinal de la Serranía del Perijá (Cesar - Colombia)
}

\section{Cesar Augusto Ruiz Agudelo}

Instituto de Ciencias Naturales-Universidad Nacional de Colombia. Apartado aéreo 7495, Bogotá, D.C. Colombia. cesarruiz1@excite.com

\section{Jaime Aguirre Ceballos}

Instituto de Ciencias Naturales-Universidad Nacional de Colombia. Programa de Doctorado en Biología- Línea de Biodiversidad y Conservación. Apartado aéreo 7495, Bogotá, D.C. Colombia.

Jaguirre@ciencias.ciencias.unaledu.co

\begin{abstract}
Resumen: De la Serranía del Perijá (Cesar-Colombia), a lo largo del gradiente altitudinal se describen cinco comunidades de briófitos (dos en las franjas subandina baja - tropical y tres en las franjas subandina baja-alta) cuyas características dependen de factores como el clima (humedad e intensidad lumínica), la estructura y la composición de la vegetación superior. Los análisis se realizaron siguiendo los lineamientos de la escuela Braun-Blanquetiana y la aplicación del Twinspan. Además, se analizan sus formas de crecimiento en todas las franjas altitudinales, las más comunes son felpas lisas, felpas rugosas e hilos.
\end{abstract}

\begin{abstract}
A long the altitudinal gradient of Serranía del Perijá (Cesar-Colombia) five communities of Bryophytes were described (two of these communities in the subandina low - tropical belt and three in the subandean low-high belt). Characteristics of these communities depend of factors such as the climate (humidity and light intensity), structure and composition on the fanerogamic vegetation. Statistical analysis followed the Braun-Blanquet school using Twinspan software. In all the altitudinal zones forms growth more common are smooth mats, rough mats, and threads.
\end{abstract}




\section{Introducción}

Los análisis de la vegetación criptogámica han tenido una larga trayectoria en las zonas templadas con descripciones cuantitativas de comunidades de briófitos epífitos de estas regiones (Barkman 1958, Hale 1955, Iwatsuki 1960, Pike et al 1975, Sjögren 1961, Wilmanns 1962). Richards (1954, 1991) describió un número importante de comunidades para los bosques de Guyana. Descripciones recientes y detalladas de comunidades de briófitos se encuentran en Cornelissen \& Steege (1989) y Montfoort \& Ek (1990), también para Guyana y Guyana Francesa.

Wolf (1993) describió las comunidades (montano bajas y montano altas) de epífitos no vasculares en un transecto altitudinal en el norte de los Andes de Colombia, analizó variables ambientales como intensidad de luz y grado de humedad.

Contribuciones sobre la distribución de briófitos epífitos han tenido mayor énfasis en las zonas templadas y subtropicales (Barkman 1958, Iwatsuki 1960, Pike et al 1975, Slack 1976, Smith 1982 , Studlar 1982, Beever 1984). En contraste, los aportes en los bosques tropicales, incluidos los de tierras bajas, son reducidos (Cornelissen \& Steege 1989).

Los briófitos epífitos se han estudiado de acuerdo con su distribución vertical desde la base del forofito hasta el dosel y la distribución horizontal, relación entre forofito y epífito (Cornelissen \& Steege 1989).

La distribución vertical ha sido tratada por Richards (1954, 1984), Florschutz-de Waard \& Bekker (1987). Pócs (1982) propuso cuatro hábitats o zonas según la altura del forofito (base, tronco, ramas bajas y ramas terminales) y concluye que la composición de especies y formas de vidas de estos hábitats es muy similar en los bosques tropicales. Cornelissen y Steege (1989), propusieron seis zonas según la altura del forofito (base- en el suelo-, tronco bajomenos de dos metros del suelo-, tronco alto- más de dos metros del suelo-, ramas bajas-primeras ramas del árbol-, ramas medias y ramas terminales-que hacen parte del dosel-) y dividieron las preferencias de las especies de briófitos epífitos en „Especialistas“ (especies que presentan un estrecho margen de distribución en diferentes hábitats, con el $90 \%$ de los registros para uno o máximo dos hábitats) y „Generalistas“ (especies con un amplio rango de distribución en los diferentes hábitats).

Como se observa, las contribuciones hasta la fecha publicadas no se ocupan de la posible relación de comunidades de briofitos y comunidades de fanerogamas. En algunos casos se insinúa su posible relación, pero nunca se ha analizado con mayor profundidad.

En la presente contribución se describen las comunidades de briófitos y su relación con los tipos de vegetación vascular en donde se establecen, a lo largo del gradiente altitudinal de la Serranía del Perijá (Cesar - Colombia). Además, se analizan las formas de crecimiento de los briófitos en dichas comunidades.

\section{METODOLOGÍA}

La descripción base del enfoque se encuentra en Aguirre \& Ruiz (2001).

Para definir las comunidades de briófitos se utilizó el programa Twinspan (Hill 1979); las tablas finales se organizaron manualmente y se ordenaron siguiendo los lineamientos de la escuela Sigmatista. (Braun-Blanquet 1979).

En los análisis de las comunidades se incluyeron los taxa que se determinaron al nivel de especie. Especies de los géneros Plagiochila y Lejeunea, no se incluyen en los análisis.

La presencia, cobertura y preferencia de las especies de briófitos sobre los diferentes tipos de habitats (zonificación, base de tronco a dosel) se estudió según Cornelissen y Steege (1989).

El estudio de las formas de crecimiento se realizó de acuerdo con Mägdefrau (1982), Studlar (1982), Richards (1984), Cornelissen \& Steege (1989) y Churchill \& Linares (1995). Para la zona de estudio se tomaron en cuenta las 
siguientes: Cespitosas bajas (Cc), Cespitosas altas (Ca), Cojines (Coj), Hilos o fibras (Hi), Felpas lisas (Fl), Felpas rugosas (Fr), Horizontales (H), Taloides (T), Péndulas (P), Dendroides no dorsiventrales (A) y Formas delgadas (B).

\section{RESULTADOS Y DISCUSIÓN}

Análisis de la vegetación (Tabla 1): Se definieron cinco comunidades de briófitos para la región del Perijá (Tabla 1), distribuidas en dos grupos de acuerdo con la altitud: A)- Compuesto por dos comunidades ( 1 y 5), por debajo del nivel de los $1500 \mathrm{~m}$ (franja subandina baja y tropical) y B)- Integrado por tres comunidades (2, 3 y 4 ) entre $\operatorname{los} 1000$ y $2000 \mathrm{~m}$ (franjas subandina baja y alta). La diferenciación obtenida en grupos de comunidades por rangos altitudinales, es similar a los valores obtenidos por Grubb (1974) y Wolf (1993).

Especies de amplia distribución (Tabla 1): Comunes en todo el gradiente altitudinal y presentes en todas las comunidades de briófitos descritas.

Lophocolea bidentata, Syrrhopodon prolifer, Telaranea nematodes y Metzgeria decipiens son de amplia distribución, con valores de presencia y cobertura que oscilan entre 20-3.8 a 100-28.3\%. Estas especies presentan diversos tipos de formas de crecimiento, entre ellas hilos (Hi), la más común en los bosques tropicales (Cornelissen \& Steege 1989); además, son generalistas, con los mayores valores de frecuencia en los hábitats base de tronco y corteza.

Vegetación criptogámica de la región Subandina baja y Tropical (<1500 m) (Tabla 1).

\section{Comunidad de Mastigolejeunea auriculata- Sematophyllum galipense}

Composición: La integran 42 especies (20 hepáticas y 22 musgos). Las exclusivas son: Mastigolejeunea auriculata, Sematophyllum galipense, Octoblepharum albidum y Drepanolejeunea subdissitifolia. Se diferenciaron 11 especies electivas de las cuales Lophocolea martiana y Drepanolejeunea subdissitifolia presentan los mayores valores de presencia y cobertura.

De las 27 especies acompañantes, Lejeunea flava y Syrrhopodon gaudichaudii son las más representativas.

Ecología y distribución: La comunidad se encuentra entre $1020 \mathrm{~m}$ (mayores valores de presencia y cobertura) y $1380 \mathrm{~m}$. Se establece en vegetación fanerogámica de tipo selvático con un estrato arbóreo superior con altos valores de área basal y cobertura. En este tipo de vegetación, la comunidad prefiriere las bases de troncos, corteza baja y troncos caídos descompuestos. Las especies fanerógamicas dominantes son Acalypha diversifolia, Adenocalymna inundatum, Amyris simplicifolia, Aphelandra pulcherrima, Bauhinia aculeata, Capparis baducca, Casearia praecox, Cecropia sp., Coccoloba candolleana, Erythroxylum cf. citrifolium, Eugenia sp., Guazuma ulmifolia, Hura crepitans, Hymenaea courbaril, Jacaranda caucana, Meliaceae sp., Neea sp., Piper amalaya, Terminalia amazonia, Throphis aff. caucana. (Arellano 2001).

El $92 \%$ de las especies de briófitos, de la comunidad, son generalistas; sólo Drepanolejeunea subdissitifolia (epifila), Leucodon curvirostris (troncos caídos descompuestos) y Sematophyllum subsimplex (troncos caídos descompuestos) son especialistas de un hábitat.

Predominan las formas de crecimiento felpas lisas (Fl), felpas rugosas (Fr) e hilos (Hi).

\section{Comunidad de Macrocoma tenue- Entodontopsis leucostega}

Composición: Está constituida por 12 especies (una hepáticas y 11 musgos). Las exclusivas son Macrocoma tenue y Entodontopsis leucostega. Se diferencian cinco especies electivas de las cuales Barbula indica (100\% presencia y $60.7 \%$ cobertura) y Fissidens mollis (100\% - 32.5\%) presentan los mayores valores de presencia y 
cobertura en la comunidad. De las 5 especies acompañantes, Calymperes lonchophyllum (50\% presencia-50\% cobertura) y Thuidium peruvianum $(50 \%-50 \%)$ presentan los mayores valores de presencia y cobertura.

Ecología y distribución: Entre 580 m (Franja tropical) y $1230 \mathrm{~m}$ (Franja subandina baja) con preferencia a $1230 \mathrm{~m}$ (mayores valores de presencia y cobertura). Se establece en varios tipos de vegetación fanerogámica, desde los bosquetes residuales de las orillas de las quebradas secas y colinas pedregosas, hasta los bosques altos entresacados de los terrenos planos en la parte alta. Las especies dominantes son Mauria suaveolens, Anthurium fendleri, Ocotea sp., Olmedia sp., Polypodium sp., Pouteria sp., Vitis tiliaefolia (Arellano 2001).

Los taxa que hacen parte de la comunidad prefirieron los hábitats: base de tronco, corteza baja y troncos caídos descompuestos, presentan los mayores valores de presencia y cobertura en base de tronco. El $90 \%$ de las especies son generalistas. Macrocoma tenue (troncos caídos descompuestos) y Thuidium peruvianum (base de tronco) son especialistas de un hábitat. En la comunidad predominan las formas de crecimiento felpas lisas $(\mathbf{F l})$, felpas rugosas $(\mathbf{F r})$ y cespitosas bajas $(\mathbf{C c})$.

Las características de la vegetación de fanerógamas (bosquete residual de baja cobertura) permiten el establecimiento de comunidades como ésta, que se ubica en base de troncos y hábitats protegidos (que conservan más humedad) en donde dominan las formas de crecimiento postradas y cortas. Estos resultados concuerdan con las consideraciones de Birse (1957), sobre las formas que dominan en condiciones de baja humedad y alta intensidad de luz.

Vegetación criptogámica de la región Subandina. franja alta y baja (>1000 y 2000 m) (Tabla 1)

Comunidad de Calypogeia miqueli Phyllogonium fulgens
Composición: La constituyen 45 especies (22 hepáticas y 23 musgos). Las exclusivas son: Phyllogonium fulgens, Calypogeia miqueli, Bazzania gracilis, Octoblepharum cocuiensi, Isodrepanium lentulum y Pallavicina lyelli. Se diferencian 5 especies electivas de las cuales Pyrrhobryum spiniforme (100\% presencia y $20.7 \%$ cobertura) y Lepidopilum scabrisetum (100\%-19\%) presentan los mayores valores de presencia y cobertura. De las 34 especies acompañantes, Lejeunea flava y Rhacopilopsis trinitensis son las más representativas.

Ecología y distribución: La comunidad se encuentra entre $1580 \mathrm{~m}$ y $1820 \mathrm{~m}$ con preferencia a $1820 \mathrm{~m}$ (mayores valores de presencia y cobertura). Se establece en vegetación fanerogámica de tipo selva multiestratificada de la franja subandina, con un estrato arbóreo superior poco desarrollado donde predominan Pouteria sp., Sloanea brevispina y Roupala montana. El estrato arbóreo inferior presenta coberturas del $35-60 \%$ y se encuentra dominado por Sloanea brevispina, Icacinaceae sp., Pouteria sp., Eschweilera sp. y Guarea aff. glabra, entre otras. Chrysochlamys colombiana domina numéricamente en el estrato sub-arbóreo mientras que Nectandra megapodamica lo hace energéticamente. Otras especies como Mikania banisteriae, Chamaedorea pinnatifrons, Guatteria cargadero, Burseraceae sp. y Guarea sp. presentan altos índices de dominancia para el estrato (Arellano 2001).

En este tipo de vegetación fanerogámica, la comunidad prefiriere los hábitats base de tronco, corteza baja y troncos caídos descompuestos, presentando los mayores valores de presencia y cobertura en corteza baja.

En las especies que hacen parte de la comunidad (Tabla 1), predominan las formas de crecimiento felpas lisas (Fl), felpas rugosas (Fr) e hilos (Hi). Están también cojines (Coj), cespitosas altas (Ca), cespitosas bajas (Cc) y dendroides no dorsiventrales (A). Estas formas indican que las condiciones que brinda la vegetación de fanerógamas (un bosque multiestratificado con mayores porcentajes de cobertura) permiten mayor retención de humedad (Barkman 1958) 
favoreciendo estas formas erectas o semierectas (Birse 1957).

Esta comunidad cuenta con un mayor número de especies acompañantes; es de esperar que un bosque complejo (mixto) ofrece mayor diversidad de hábitats y por lo tanto se favorece la ocurrencia de más especies (Gradstein et al., 1990).

\section{Comunidad de Shymphyogyna aspera- Shymphyogyna brogniarti}

Composición: La integran 69 especies (28 hepáticas y 41 musgos). Las exclusivas (4) son: Symphyogyna aspera, Symphyogyna brogniarti, Metzgeria maegdefraui e Isotachys multiceps. Se diferencian 13 especies electivas de las cuales Thuidium tomentosum (83\% presencia y $8.3 \%$ cobertura) e Hypopterygium tamariscinum (66\%-14.45\%) presentan los mayores valores de presencia y cobertura.

De las 52 especies acompañantes, Sematophyllum subpinnatum, Mittenathamnium reptans y Neckeropsis undulata presentan los mayores valores de presencia y cobertura. Son comunes especies de los géneros Plagiochila, Lejeunea y algunas Parmeliaceas.

Ecología y distribución: La comunidad se encuentra entre $1200 \mathrm{~m}$ y $1820 \mathrm{~m}$ con preferencia a $1720-1810 \mathrm{~m}$ (mayores valores de presencia y cobertura). Se establece en vegetación fanerogámica de tipo selva multiestratificada subandina, con elementos como Billia rosea, Ficus cuatrecasae y Guarea kunthiana en el estrato arbóreo superior, con promedios de 30 y $31 \mathrm{~m}$ de altura y coberturas del $35-65 \%$. En este estrato existe un marcado predominio fisionómico y numérico de $G$. kunthiana. El estrato arbóreo inferior está dominado por Prunus integrifolia y Ficus cuatrecasae seguidos de Persea sp., Machaerium sp. y Nectandra sp. En los estratos sub-arbóreo y arbustivo dominan Piper medium, Parathesis adenanthera, Persea sp. y Ficus sp. con algunas variaciones de posición dentro de éstos. Se encontraron especies epífitas como Peperomia tetraphylla, Pleurothallis sp., Tillandsia complanata, Govenia sp. y trepadoras como Amphilophium paniculatum, Blepharodon sp., Convolvulaceae sp., Fevillea cordifolia, Macfadyena unguis-cati, Malvaviscus elegans y Mikania banisteriae (Arellano 2001).

Esta comunidad se establece en tipos de vegetación fanerogámica similares (estructuralmente) a los de la comunidad de Calypogeia miqueli-Phyllogonium fulgens. Las especies de esta comunidad prefirieren los hábitats base de tronco, corteza baja y troncos caídos descompuestos, con los mayores valores de presencia y cobertura en corteza baja. El 96\% de las especies son generalista. Isotachys multiceps es exclusiva del hábitat suelo (próximo a base de troncos). Predominan las formas de crecimiento felpas lisas $(\mathbf{F l})$, felpas rugosas $(\mathbf{F r})$ e hilos (Hi).

Están bien representadas las formas: cojines (Coj), cespitosas altas (Ca), cespitosas bajas (Cc), talosas (T) y dendroides no dorsiventrales (A).

\section{Comunidad de Neckera scabridens - Papillaria nigrescens}

Composición: La constituyen 74 especies (29 hepáticas y 45 musgos). Son exclusivas Neckera scabridens, Papillaria nigrescens, Prionodon fusco-lutescens, Eurhynchium semiscabrum, Rhynchostegium serrulatum, Lopholejeunea nigricans, Papillaria imponderosa, Fissidens serratus y Marchantia polymorpha. Se diferencian seis especies electivas de las cuales Prionodon densus (100\% presencia y $9.86 \%$ cobertura) y Porotrichum lindigii (80\% - 9.7\%) presentan los mayores valores de presencia y cobertura.

De las 59 especies acompañantes, Sematophyllum subpinnatum $(80 \%$ presencia$8.15 \%$ cobertura) y Mittenathamnium reptans (80\%-7.65\%) son las más representativas.

Ecología y distribución: Esta comunidad es exclusiva de la región de vida subandina alta. Se encuentra entre $1750 \mathrm{~m}$ y $2000 \mathrm{~m}$ con preferencia a $1950 \mathrm{~m}$ (mayores valores de presencia y cobertura). Se establece en vegetación fanerogámica de tipo selva subandina, con cinco estratos bien diferenciados y con altos porcentajes de cobertura (45-65\%). 
En el estrato arbóreo inferior el predominio fisionómico es de Ficus sp. y Nectandra sp. seguidos por Prunus integrifolia, Persea caerulea e Inga minutula. El estrato sub-arbóreo, se encuentra dominado fisionómicamente por Guettarda sp. y Piper lanceaefolium, aunque, en los valores de dominancia numérica predominan Piper lanceaefolium e Inga minutula. Se registraron, además especies epifitas como Vriezea incurva, Mikania sp., Pleurothallis sp., Cinanchum tenellum y Rhipsalis baccifera (Arellano 2001).

En este tipo de vegetación fanerogámica, la comunidad prefiriere los hábitats base de tronco y corteza baja. Están bien representados troncos caídos descompuestos, ramas bajas y suelo (próximo a las bases de tronco). El $98 \%$ de las especies son generalistas en cuanto su preferencia a hábitats.

Las formas de crecimiento que dominan en las especies que constituyen la comunidad son felpas lisas (Fl), felpas rugosas (Fr), formas péndulas (P), dendroides no dorsiventrales (A) y formas delgadas (B). La abundancia de formas péndulas y dendroides no dorsiventrales indica que las condiciones que brinda la vegetación de fanerógamas (bosques multiestratificados con buena humedad y porcentajes altos de cobertura) permiten mayor retención de humedad y favorecen el epifitismos de ramas y porciones del árbol más altas (por ejemplo la especie Neckera scabridens de habito péndulo predomina en el hábitat ramas bajas).

El establecimiento de esta comunidad en el límite de las regiones de vida subandina alta y andina, con el mayor número de especies (74), corrobora que la diversidad de briófitos aumenta a medida que aumenta la altura sobre el nivel del mar (Churchill \& Linares 1995, Aguirre \& Ruiz 2001). Es claro que las características de las comunidades de briófitos epífitos depende de los factores características del bosque de fanerógamas, humedad ambiental e intensidad de luz (Wolf 1993).

Elementos atípicos: Se mencionan a continuación los elementos (levantamientos) atípicos (aislados), que no hacen parte de ninguna comunidad ya que su composición briflorística es muy distinta.

Comunidad de Styrax aff. pseudoargyrophylla y Ocotea sp. (Fernández Jl. 13084): Municipio: Jagua de Ibirico, Alto de las flores, Finca el Paraíso, Franja subandina baja, (1020-1590), Altitud $1420 \mathrm{~m}-1580 \mathrm{~m}$.

La constituyen 20 especies de briófitos (11 especies de musgos y nueve de hepáticas). Lepidopilum scabrisetum, Isopterygium tenerum, Pyrrhobryum spiniforme y Thuidium tomentosum presentan los mayores porcentajes de cobertura (25.0 a $26.0 \%$ ). Especies de los géneros Plagiochila, Lejeunea y Leptogium se encuentran bien representadas.

Los briófitos se establecen en vegetación fanerogámica del tipo selva multiestratificada de la franja subandina, con un estrato arbóreo superior, donde dominan especies de Pouteria sp., Ocotea sp., Billia columbiana, Hirtella triandra y Sloanea brevispina entre otras, el cual presenta alturas promedio de 27 a $32 \mathrm{~m} \mathrm{y}$ porcentajes medios de cobertura. En el estrato arbóreo inferior predominan Pouteria sp., Heliocarpus aff. americanus, Billia rosea, Dendropanx arboreus e Hirtella triandra. Especies como Psychotria deflexa, Nectandra aff. acutifolia, Heliocarpus americanus, Dendropanax arboreus dominan el estrato subarbóreo, mientras que Ficus sp., Nectandra aff. acutifolia, Mauria suaveolens y Piper obliquum, lo hacen para el arbustivo (Arellano 2001).

\section{Asociación Piperimedium-Lozanelletum enantiophyllae}

Comunidad de Opuntia aff. schumanni y Xylosma obovatum : Agustín Codazzi, Vereda ara de Hombre, Finca Alsides, franja subandina alta.(1600 a $2000 \mathrm{~m})$. Altitud $1860 \mathrm{~m}$.

Syrrhopodon gaudichaudii y Fissidens aspleniodes, son las únicas especies de briófitos, con coberturas del 25\%. Estas especies de briófitos, se establecen en vegetación fanerogámico de tipo Cardonal azonal, biestratificado, perteneciente a la franja 
subandina, los estratos herbáceo y rasante presentan predominio fisionómico de Opuntia shumanni, Xylosma obovatum y Verbesina aff. lloensis. Además, los estratos presentan valores de cobertura del 10 al 15\% (Arellano 2001).

Las características que exhibe este tipo de vegetación (cardonal), no favorecen el establecimiento y desarrollo de las especies de briófitos.

Formas de crecimiento (Figura 1): Felpas lisas (FL, 39 especies) y felpas rugosas (Fr, 23 especies) son las formas de crecimiento dominantes y las más exitosas en las condiciones ambientales (alta temperatura, alta intensidad de luz y baja precipitación <2000 mm/año) de la Serranía del Perijá (Aguirre \& Ruiz 2001). Richards (1984), Cornelissen \& Steege (1989) y Gradstein et al. (1990) ya habían expuesto este comportamiento al presentar resultados similares para otros bosques tropicales y de zonas templadas.

Las formas de crecimiento menor representadas son: Cespitosas altas (Ca, $6 \mathrm{sp})$ y cojines $(\mathbf{C o j}$, $4 \mathrm{sp})$.

\section{CONSIDERACIONES FINALES}

En la serranía del Perijá se distinguen dos grupos de comunidades según rangos altitudinales: Comunidades de las franjas Subandina baja y Tropical y comunidades de las franjas Subandina alta y baja.

El establecimiento de las comunidades de briófitos en los diferentes hábitats, depende de la naturaleza del bosque de fanerogámas, de la humedad ambiental y de la intensidad de luz.

En la serranía del Perijá la mayor parte de las comunidades (sin importar la franja altitudinal) se ubican, con preferencia, en base de tronco y corteza baja, situación que tiene directa relación con la baja humedad y la estructura del bosque de fanerogámas.

Las formas de crecimiento son un indicativo directo de la naturaleza de las comunidades, ya que en cada una predomina una forma en particular que indica la respuesta de las especies a los factores ambientales.

Según Gradstein et al. (1990) los bosques mixtos y complejos (multiestratificados) ofrecen una mayor diversidad de hábitats para que se establezca una mayor diversidad de especies y de comunidades de briófitos. Esta situación se observa en la serranía del Perijá, ya que en los bosques más complejos de la franja subandina alta, ocurren las comunidades más diversas tanto en especies como en formas de crecimiento.

En los bosques de la Serranía del Perijá las especies de briófitos muestran un patrón de distribución en los diferentes hábitats.

Las formas de crecimiento dan una idea de las estrategias de las especies para sacarle el mejor provecho a un determinado hábitat (Cornelissen \& Steege 1989, Gradstein et al. 1990). Éstas varían mucho en la Serranía del Perijá, pero siempre sin importar la comunidad corteza baja, es el habitat más común. .

\section{Literatura citada}

Aguirre J. \& C.A. Ruiz. 2001. Composición florística de la brioflora de la Serranía de Perijá (Cesar - Colombia): Distribución y ecología. Caldasia 23(1): 181-201.

Arellano, H. 2001. Análisis de la vegetación del sistema andino de la Serranía de Perijá (Cesar-Colombia). Tesis degrado. Universidad Nacional de Colombia Instituto de Ciencias Naturales. Bogotá D.C.

Barkman, J. 1958. Phytosociology and ecology of cryptogamic epiphytes. Van Gorcum. Assen. 628 pp.

Beever, J.E. 1984. Moss epiphytes of tree-ferns in a warm-temperate forest, New Zealand, Journal Hattori Botanical Laboratory 56: 89-95.

Birse, E.M. 1957. Ecologycal studies on growthform in bryophytes. II Experimental studies on growth-form in mosses. Journal of Ecology 45: 721-733. 
Braun-Blanquet, J. 1979. Fitosociología: bases para el estudio de las comunidades vegetales. Ed. Blume, Madrid.

Churchill, S.P. \& E.L. Linares. 1995. Prodomus Bryologiae Novo-Granatensis. Introducción a la flora de musgos de Colombia. Biblioteca José Jerónimo Triana. Tomos I y II. Instituto de Ciencias Naturales. Facultad de Ciencias. Universidad Nacional de Colombia. Bogotá.

Cornelisen, J.H.C \& H.T, Steege. 1989 . Distribution and ecology of epiphytic bryophytes and lichens in dry evergreen forest of Guyana. Journal of Tropical Ecology 5:131-150.

Florscutz-de Waard, J. \& J.M, Bekker. 1987. A comparative study of the bryophyte flora of different forest types in West Surinam. Cryptogamie, Bryologie Lichenologie 8:31-45.

Gradstein, S.R. 1995. Bryophyte diversity of the tropical rainforest. Archs. Sci. Geneve 48(1):91-95.

Gradstein, S.R., D. Montfoort \& J.H.C. Cornelissen. 1990. Species richness and phytogeography of the bryophyte flora of the Guianas, with special reference to the lowland forest. Tropical Bryology 2:117126.

Grubb, P.J. 1974. Factors controlling the distribution of forest-types on tropical mountains: new facts and a new perspectives. En. Flenley, J.R. (ed). Altitudinal zonation in Malesia. Univ. of Hull. Misc. Ser. 16:13-45.

Hale, M.E. 1955. Phytosociology of corticolous cryptogams in the upland forest of southern Wisconsin. Ecology 36:4563.

Hill, M.O. 1979. TWINSPAN. A FORTRAN program for arranging multivariate data in a ordered two-way table by classification of the individuals and attributes. Cornell University, Ithaca.

Iwatsuki, Z. 1960. The epiphytic bryophyte communities in Japan. Journal Hattori Botanical Laboratory 22: 159-348.

Kürschner, H. 1990. Die epiphytischen Moosgesellschaften am Mt. Kinabalu (Nord- Borneo, Sabah, Malaysia). Nova Hedwigia 51: 1-75.

Mägdefrau, K. 1982. Life - forms of bryophytes. Pp. 45-58 in: Smith, A.J.E. (ed).Bryophyte ecology. Chapman and Hall.

Montford, D. \& Ek, R. 1990. Vertical distribution and ecology of epiphytic bryophytes and lichens in a lowland rain forest of French
Guiana. M. Sc. Thesis, University of Utrecht.

Pike, L. H. Denison, W.C., Tracy, D.M., Sherwood, M.A. \& F.M. Rhoades. 1975. Floristic survey of epiphytic lichens and bryophytes growing on old growth conifers in Western Oregon. Bryologist 78: 389-402.

Pócs, T. 1982. Tropical forest bryophytes. Pp. 4558. En Smith, A.J.E. (ed). Bryophyte ecology.

Rangel-CH, J.O. 1996. Comunidades vegetales del sector de la Jagua de Ibirico, Serranía del Perijá. Informe convenio cooperación CORPOCESAR-Universidad Nacional de Colombia-Instituto de Ciencias Naturales (Inedito.).

Richards, P.W. 1954. Notes on the bryophyte communities of lowland tropical rain forest, with special reference to Moraballi creek, British Guyana. Vegetatio 5-6:319-328.

Richards, P.W. 1984. The ecology of tropical forest bryophytes. En: Schuster, R.M. (ed). New manual of bryology 2: 1233-1270.

Richards, P.W. 1991 . A bryologist in British Guiana and the West Indies. J. Bryol. 16:437-441.

Slack, N.G. 1976. Host specifity of bryophytic epiphytes in Eastern North America. Journal Hattori Botanical Laboratory 41: 107-132.

Smith, A.J.E. 1982. Epiphytes and epiliths Pp. 191227 en: Smith, A.J.E (ed). Bryophyte ecology.

Sjögren, E. 1961. Epiphytische Moosvegetation in Laubwäldern der Insel Öland. Acta Phytogeogr. Suec. 44:1-149.

Söderstron, 1. 1993. Substrate preference in some forest bryophytes: a quantitative study. Lindbergia 18: 98-103.

Studlar, S.M. 1982. Succession of epiphytic bryophytes near Mountain Lake, Virginia. Bryologist 85: 51-63.

Wilmanns, O. 1962. Rindenbewohnende Epiphytengeminschaften in Südwestdeutschland. Beitr. Naturk. Forsch. SW-Deutschl. XXI(2):87-164.

Wolf, H.D. 1993. Epiphyte communities of tropical montane rain forest in the northern Andes. I. Lower montane communities. Phytocoenologia 22(1): 1-52. 


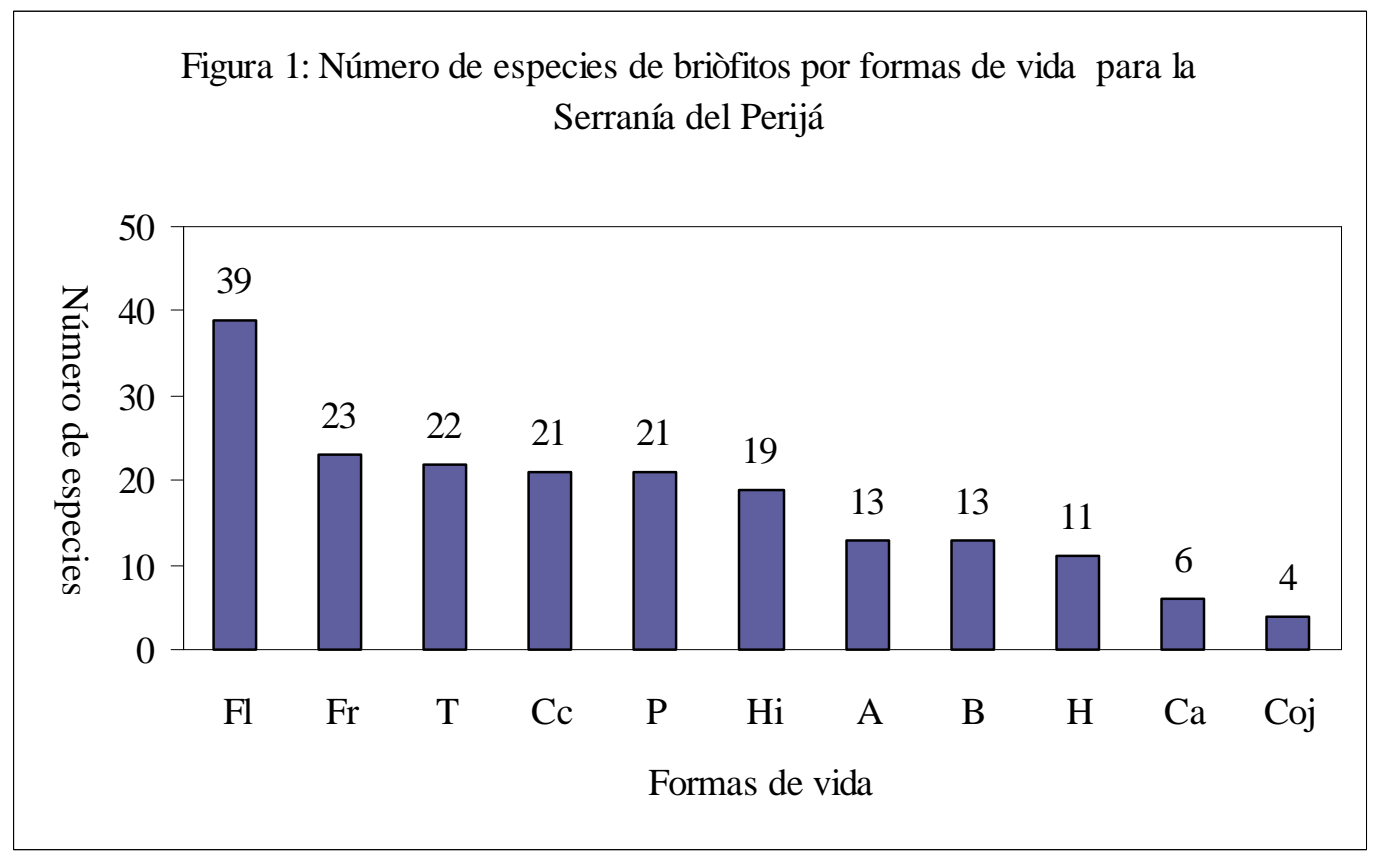

TROPICAL BRYOLOGY 24 (2003) 
Tabla 1:

* Especies exclusivas

+ Especies electivas
Cuadro sinóptico de las comunidades de briófitos de la Serranía del Perijá

con base en porcentajes de presencia y cobertura

\begin{tabular}{|c|c|c|c|c|c|c|c|c|c|c|}
\hline Comunidad & 1 & 1 & 2 & 2 & 3 & 3 & 4 & 4 & & 5 \\
\hline $\begin{array}{l}\text { No. de levantamientos de la } \\
\text { comunidad }\end{array}$ & 2 & & 3 & & 6 & & 5 & & 2 & \\
\hline Franja altitudinal & $\begin{array}{l}\text { SF } \\
\text { B }\end{array}$ & $\begin{array}{l}2 \\
\text { Lev }\end{array}$ & $\begin{array}{l}\text { SF } \\
\text { A } \\
\text { SF } \\
B\end{array}$ & $\begin{array}{l}2 \text { Le } \\
v \\
1 \\
\text { Lev }\end{array}$ & $\begin{array}{l}\mathrm{SF} \\
\mathrm{A} \\
\mathrm{SF} \\
\mathrm{B}\end{array}$ & $\begin{array}{l}5 \\
\text { Lev } \\
1 \text { Le } \\
v\end{array}$ & $\begin{array}{l}\text { SF } \\
\text { A }\end{array}$ & $\begin{array}{l}5 \\
\text { Lev }\end{array}$ & $\begin{array}{l}\text { SF } \\
B \\
\text { TR } \\
0\end{array}$ & $\begin{array}{l}1 \text { Le } \\
v \\
1 \mathrm{Le} \\
\mathrm{v}\end{array}$ \\
\hline \multirow{3}{*}{$\begin{array}{l}\text { No. Total de taxas en la } \\
\text { comunidad }\end{array}$} & 42 & & 45 & & 69 & & 74 & & 12 & \\
\hline & pres & cober & pres & cober & pres & cober & pres & cober & pres & cober \\
\hline & $\%$ & $\%$ & $\%$ & $\%$ & $\%$ & $\%$ & $\%$ & $\%$ & $\%$ & $\%$ \\
\hline
\end{tabular}

Amplia distribución Lophocolea bidentata Syrrhopodon prolifer Telaranea nematodes Metzgeria decipiens

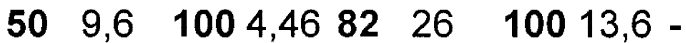

- $\quad 1006,68316205,2$.

- $\quad 10028,3667,3403,8$

- $\quad$ - $\quad 1003,56837,36407,65$.

1 Comunidad de Mastigolejeunea auriculata-Sematophyllum galipense

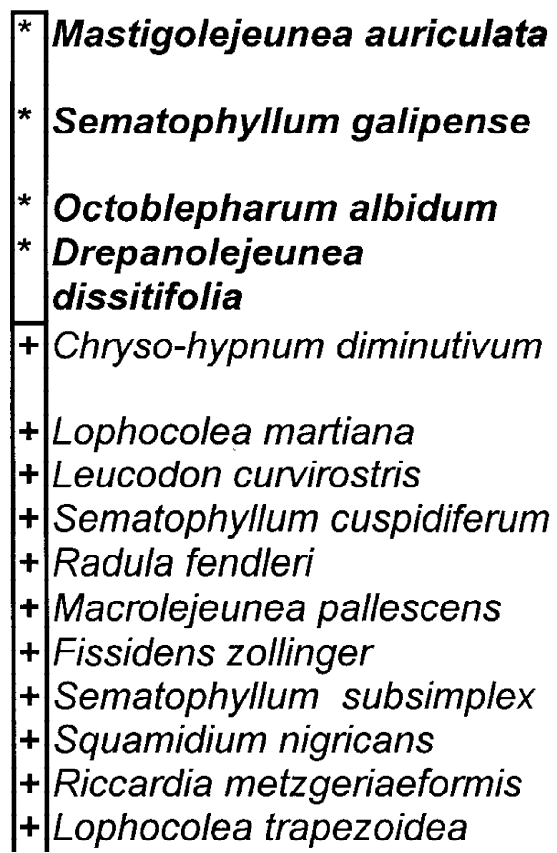

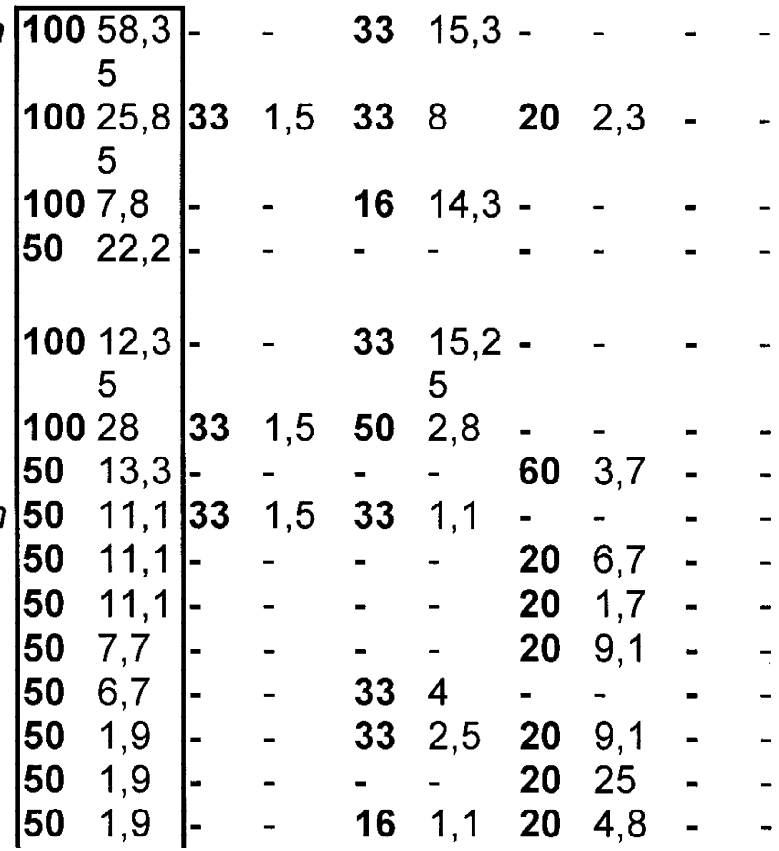


2 Comunidad de Calypogeia miqueli Phyllogonium fulgens

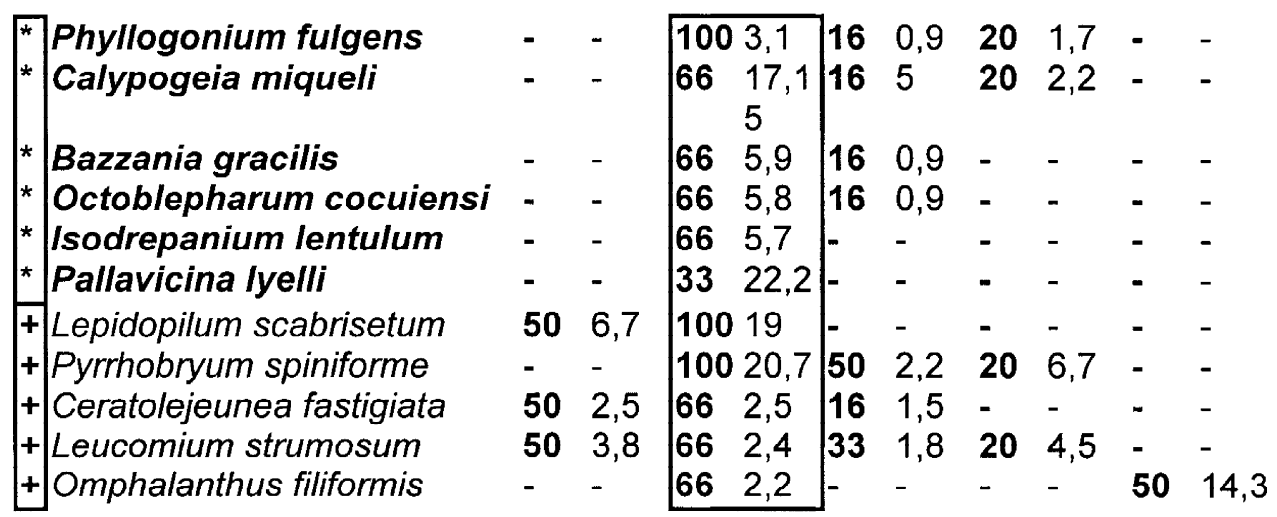

3 Comunidad de Symphyogyna aspera - Symphyogyna brogniarti

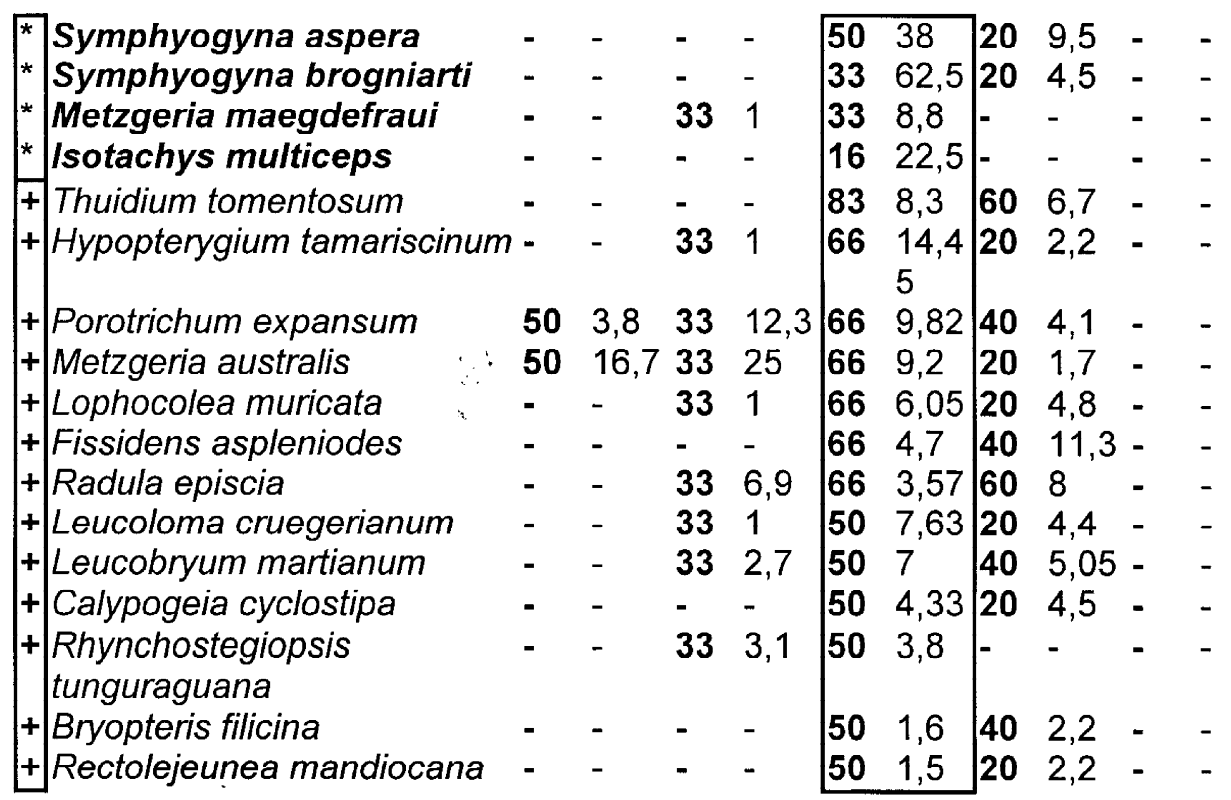

\section{Comunidad de Neckera scabridens - Papillaria nigrescens}

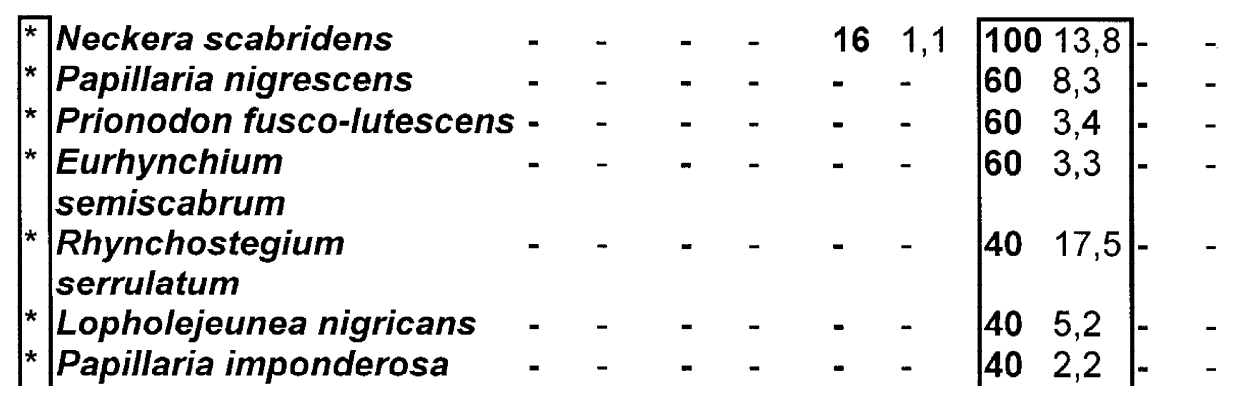

TROPICAL BRYOLOGY 24 (2003) 


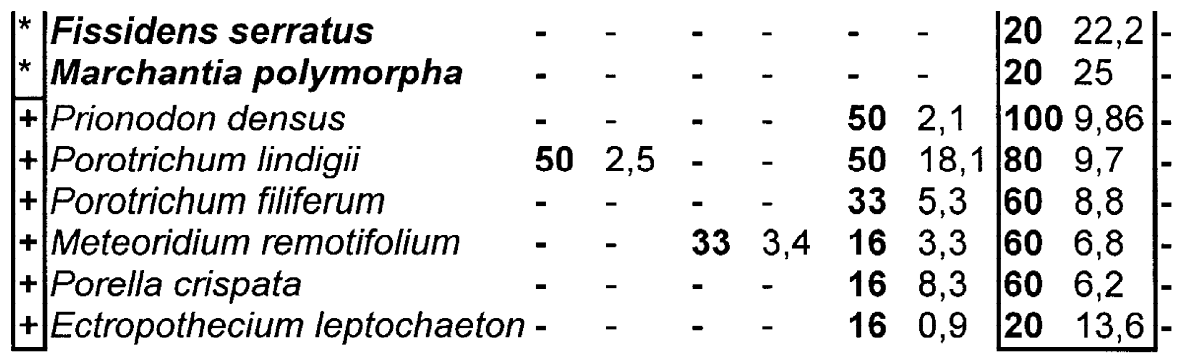

\section{Comunidad de Macrocoma tenue - \\ Entodontopsis leucostega}

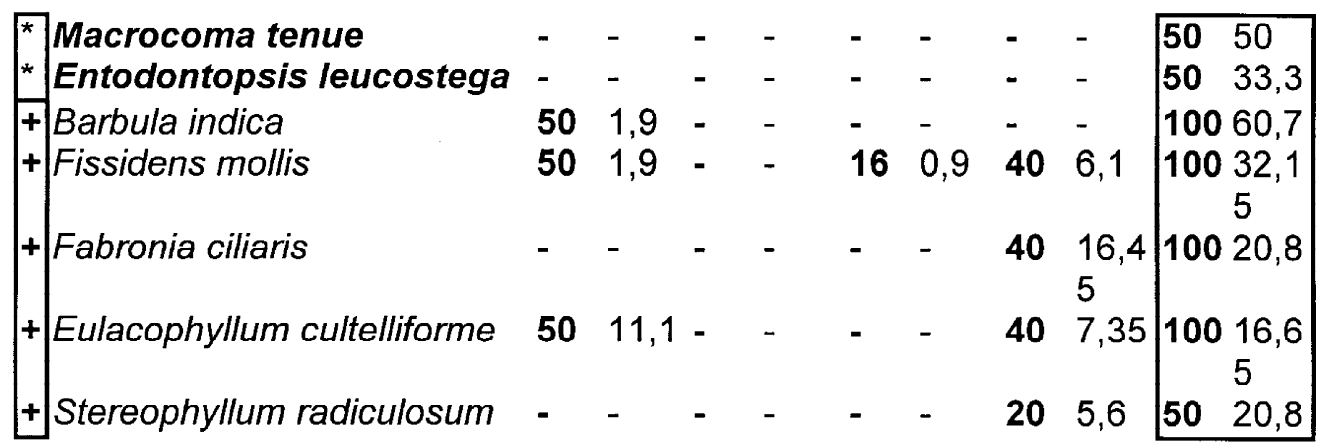

Acompañantes con preferencia a la comunidad uno

Lejeunea flava

Syrrhopodon gaudichaudii Drepanolejeunea inchoata Monoclea gottschei

Dumortiera hirsuta

Acompañantes con comunidades dos y tres

Rhacopilopsis trinitensis

Metzgeria leptoneura

Calypogeia peruviana

Arachniopsis diacantha

Bazzania affinis

Calymperes lonchophyllum

Leptolejeunea elliptica

Trachyxiphium subfalcatum

Pilotrichella flexilis

Radula sinuata

Lepidozia wallisiana

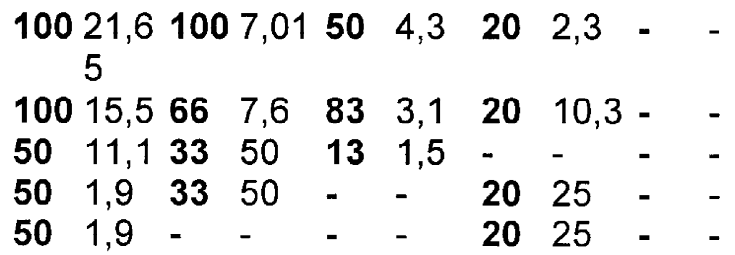

preferencia a las

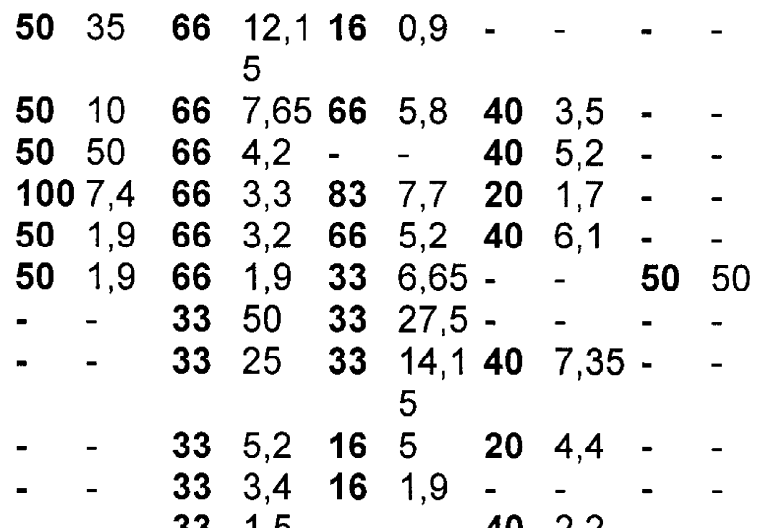


Acompañantes con preferencia a las comunidades tres y cuatro

Sematophyllum subpinnatum - $\quad$ - $\quad$ - $\quad$ - $10010,6808,15$ - $\quad$ -

6

Mittenothamnium reptans $\quad$ - $\quad-\quad 33 \quad 5,2 \quad 1007,2 \quad 80 \quad 7,65$ -

Neckeropsis undulata $\quad 1002,2 \quad-\quad$ - $8325,6206,7$

Microlejeunea bullata $\quad 5066,7$ - $\quad 663,3205,2$ - $\quad$ -

Rhynchostegium scariosum - $\quad$ - $\quad 508,836011,9$ - $\quad$ -

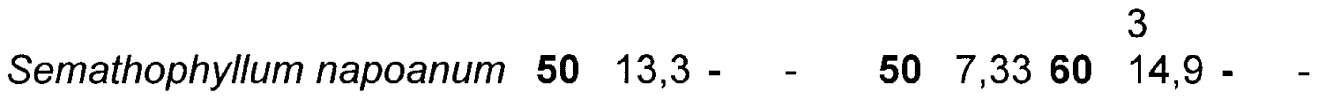

$\begin{array}{lllllllllll}\text { Isopterygium tenerifolium } & \mathbf{5 0} & 6,7 & 33 & 3,7 & \mathbf{5 0} & 2,73 & - & - & - & -\end{array}$

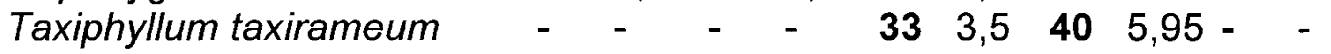

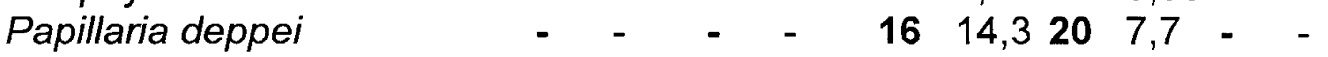

Calyptothecium duplicatum - $\quad$ - $\quad$ - $\quad$ - 16540565 -

Porella swartziana $\quad 5016,7$ - $\quad$ - $\quad$ - $\quad$ - $\quad 40 \quad 12,5 \mathbf{5 0} 18,2$

Racopilum tomentosum $\quad 5011,1$ - $\quad$ - $\quad$ - 805,6 - -

Acompañantes con preferencia a la comunidad cinco

$\begin{array}{lllllllllll}\text { Thuidium peruvianum } & - & - & - & - & 33 & 1,2 & 40 & 1,9 & \mathbf{5 0} & 50\end{array}$

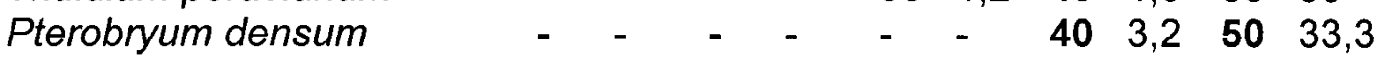

Accidentales

Rhvnchosteaiopsis flexuosa - $\quad$ - $\quad$ - $\quad 16 \quad 5 \quad 203.4$ - - 
